\title{
CUSP ALGEBRAS
}

\author{
Jim AgleR* AND John E. MCCARTHY ${ }^{\dagger}$
}

Abstract

A cusp is the image of the unit disk under a proper holomorphic map into $\mathbb{C}^{n}$ that is one-to-one and whose derivative vanishes at exactly one point. It is simple if not all the second derivatives vanish. We characterize when two simple cusps are isomorphic, and show that they can all be realized in $\mathbb{C}^{2}$.

\section{Introduction}

By a cusp $V$ we shall mean the image of the unit disk $\mathbb{D}$ under a bounded injective holomorphic map $h$ into $\mathbb{C}^{n}$ whose derivative vanishes at exactly one point. The simplest example is the Neil parabola, given by $h(\zeta)=\left(\zeta^{2}, \zeta^{3}\right)$. See $[\mathbf{3}],[\mathbf{4}]$ for background and theory on the Neil parabola, which is pictured in Figure 1.

A generalization of a cusp is a petal. A petal is the image of the unit disk $\mathbb{D}$ under a proper holomorphic map $h$ from $\mathbb{D}$ into some bounded open set $\Omega$, where, except for a finite set $E_{h}, h$ is one-to-one and nonsingular. If $E_{h}$ is a singleton, then $V$ is a cusp. Since the automorphism group of $\mathbb{D}$ is transitive, we may assume then that $E_{h}=\{0\}$.

The function $h: \mathbb{D} \rightarrow \Omega$ induces a finite codimensional subalgebra $A_{h}$ of the algebra $O(\mathbb{D})$ of all analytic functions on $\mathbb{D}$, namely

$$
A_{h}=\{F \circ h: F \in O(V)\},
$$

where $O(V)$ is the algebra of functions on $V$ that, in the neighborhood of every point $P$ of $V$, coincides with the restriction to $V$ of a function holomorphic in a neighborhood of $\mathbb{C}^{n}$ containing $P$. When $V$ is a cusp, the algebra $A_{h}$ has the property that it contains $z^{m} O(\mathbb{D})$ for some $m \geq 2$. Conversely, if a cofinite subalgebra of $O(\mathbb{D})$ contains $z^{m} O(\mathbb{D})$, then it arises in this way.

2000 Mathematics Subject Classification. 14H10, 30F20.

Key words. Petal, cusp, holomap.

* Partially supported by National Science Foundation Grant DMS 0400826.

${ }^{\dagger}$ Partially supported by National Science Foundation Grant DMS 0501079. 


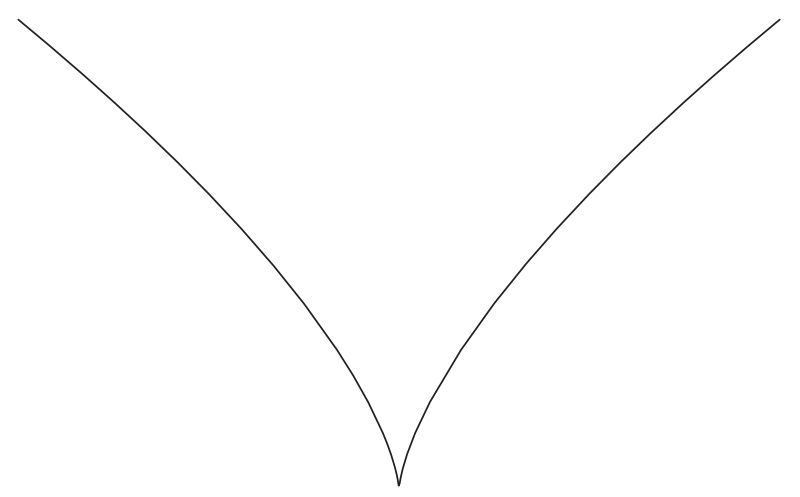

Figure 1. A simple cusp - the Neil parabola $z^{3}=w^{2}$.

In light of these remarks, let us agree to say that a unital algebra $A \subseteq$ $O(\mathbb{D})$ is a cusp algebra if

(i) $\operatorname{dim}(O(\mathbb{D}) / A)$ is finite, and

(ii) for some $m \geq 2, z^{m} O(\mathbb{D}) \subseteq A$.

We shall say a map $h: \mathbb{D} \rightarrow \Omega \subsetneq \mathbb{C}^{n}$ is a holomap if it is a proper map that is one-to-one and non-singular except on a finite set. We shall say that a holomap $h$ is a holization of the cofinite algebra $A$ if $A=A_{h}$.

If $A$ is a cusp algebra, then we define three integers attached to $A$. The codimension of $A$ is defined by

$$
\operatorname{cod}(A)=\operatorname{dim}(O(\mathbb{D}) / A) .
$$

The order of $A$ is defined by

$$
\operatorname{ord}(A)=\min \left\{k: z^{k+1} O(\mathbb{D}) \subseteq A\right\} .
$$

The contact of $A$ is defined by

$$
\operatorname{con}(A)=\max \left\{n: f^{(j)}(0)=0, \forall f \in A, 1 \leq j \leq n\right\} .
$$

If $V$ is a cusp, then we define $\operatorname{cod}(V), \operatorname{ord}(V)$ and $\operatorname{con}(V)$ as the quantities for the corresponding algebra $A_{h}$.

By a simple cusp algebra, we shall mean a cusp algebra $A$ such that $\operatorname{con}(A)=1$. There is a unique cusp algebra of codimension 1 , which corresponds via a holization to the holomorphic functions on the Neil parabola $\left\{(z, w) \in \mathbb{D}^{2}: z^{3}=w^{2}\right\}$ (this result can be seen directly, or will follow from Section 4). This can be thought of as a Riemann mapping theorem for cusps of codimension 1. In Section 4, we generalize this "Riemann mapping theorem" to arbitrary simple cusps. We show that 
locally the only invariant of simple cusps is the codimension, but globally cusps of codimension $n+1$ have a $2 n-1$ real parameter moduli space.

This paper is a continuation of the authors' earlier work in [1], where the ideas are put in a more general context. A principal concern in that paper is when a given finite codimensional subalgebra can be holized in 2 dimensions, i.e. by a map into $\mathbb{C}^{2}$. In Section 5 , we show that all simple cusp algebras can be holized in $\mathbb{C}^{2}$.

\section{Preliminaries}

Throughout this section, we shall assume that $A$ is a simple cusp algebra, i.e. of contact one. By a primitive for $A$ we mean a function $\pi$ that lies in $A$, and satisfies $\pi(0)=0, \pi^{\prime \prime}(0)=2$; so $\pi$ has a Taylor expansion $\pi(z)=z^{2}+\cdots$.

For each $n$, let $A_{n}$ be the space

$$
A_{n}=\left[A \cap z^{2 n} O(\mathbb{D})\right],
$$

and let $E_{n}$ be the quotient

$$
E_{n}=A_{n} / A_{n+1} .
$$

Observe that multiplication by any primitive $\pi$ is always one-to-one from $E_{n}$ to $E_{n+1}$. Therefore, there is some integer $n_{0}$ such that $\operatorname{dim}\left(E_{n}\right)$ is 1 for $n \leq n_{0}$, and 2 for $n>n_{0}$. Since $\operatorname{dim}\left(E_{n}\right)=2$ if and only if both $z^{2 n}$ and $z^{2 n+1}$ are in $A$, it follows that

$$
\operatorname{ord}(A)=2 n_{0}+1 \text {. }
$$

As $\pi^{n}$ is in $A_{n}$ for each $n$, if $f$ is in $A_{n}$ for some $n \leq n_{0}$, then there is a constant $c_{n}$ such that $f-c_{n} \pi^{n}$ is in $A_{n+1}$. Therefore every function $f$ in $A$ has the representation

$$
f=c_{0}+c_{1} \pi+\cdots+c_{n_{0}} \pi^{n_{0}}+z^{2 n_{0}+2} g
$$

where $c_{0}, \ldots, c_{n_{0}}$ are in $\mathbb{C}$ and $g \in O(\mathbb{D})$. From $(2.1)$, we see that $\operatorname{cod}(A)=n_{0}+1$. Summarizing, we have:

Proposition 2.2. If $A$ is a simple cusp algebra, then $\operatorname{ord}(A)=2 \operatorname{cod}(A)-$ 1. Furthermore, if we set $n_{0}=\operatorname{cod}(A)-1$, and $\pi$ is a primitive for $A$, then every function $f$ in $A$ has a unique representation

$$
f(z)=p \circ \pi(z)+z^{2 n_{0}+2} g(z)
$$

for some polynomial $p$ of degree at most $n_{0}$ and some $g \in O(\mathbb{D})$. 


\section{Connections and local theory}

Let $U$ be an open set in $\mathbb{C}$. A linear functional on $O(U)$ is called local if it comes from a finitely supported distribution, i.e. is of the form

$$
\Lambda(f)=\sum_{i=1}^{m} \sum_{j=0}^{n_{i}} a_{i j} f^{(j)}\left(\alpha_{i}\right) .
$$

Definition 3.1. A connection on $\left\{\alpha_{1}, \ldots, \alpha_{m}\right\} \subset U$ is a finite dimensional set $\Gamma$ of local functionals $\Lambda$ supported by $\left\{\alpha_{1}, \ldots, \alpha_{m}\right\}$. We say $\Gamma$ is algebraic if $\Gamma^{\perp}:=\{f \in O(U): \Lambda(f)=0, \forall \Lambda \in \Gamma\}$ is an algebra.

It was proved by T. W. Gamelin $[2]$ that every finite codimensional subalgebra $A$ of $O(\mathbb{D})$ is $\Gamma^{\perp}$ for some algebraic connection on $\mathbb{D}$. Moreover, $A$ will be a cusp algebra iff the support of $\Gamma$ is $\{0\}$.

We shall say that a point $P$ in a petal $V$ is a cusp point if there is a one-to-one proper map $h$ from $\mathbb{D}$ onto a neigborhood $U$ of $P$ in $V$ such that the derivative of $h$ vanishes only at the pre-image of $P$. The algebra $A_{h}$ is then a cusp algebra. We wish to show that its codimension, order and contact do not depend on the choice of $U$.

Suppose that $P_{1}$ and $P_{2}$ are cusp points in cusps $V_{1}$ and $V_{2}$, and there is an injective holomorphic map $\phi: V_{1} \rightarrow V_{2}$. For $r=1,2$ there are holizations $h_{r}: \mathbb{D} \rightarrow V_{r}$ with $h_{r}(0)=P_{r}$. By [1, Theorem 3.2], the map $\phi$ from $V_{1}$ to $V_{2}$ induces a one-to-one map $\psi: \mathbb{D} \rightarrow \mathbb{D}$ such that $h_{2} \circ \psi=\phi \circ h_{1}$, and the connections $\Gamma_{1}$ and $\Gamma_{2}$ induced by $h_{1}$ and $h_{2}$ are related by

$$
\begin{aligned}
\Gamma_{2} & \supseteq \psi_{*} \Gamma_{1} \\
& :=\left\{f \mapsto \Lambda_{1}(f \circ \psi): \Lambda_{1} \in \Gamma_{1}\right\} .
\end{aligned}
$$

If

$$
\Lambda_{1}: f \mapsto \sum_{j=0}^{n} a_{j} f^{(j)}(0),
$$

then

$$
\psi_{*} \Lambda_{1}: g \mapsto \sum_{j=0}^{n} a_{j}(g \circ \psi)^{(j)}(0) .
$$

We can use Faá di Bruno's formula to evaluate the derivatives of $g \circ \psi$ in terms of those of $g$ and $\psi$. As $\psi(0)=0$ and $\psi^{\prime}(0) \neq 0$, note in particular that the coefficient of $g^{(n)}(0)$ on the right-hand side of $(3.3)$ is $a_{n}\left[\psi^{\prime}(0)\right]^{n}$. Therefore $\psi_{*}$ is injective, so $\operatorname{cod}\left(A_{1}\right) \leq \operatorname{cod}\left(A_{2}\right)$.

If $\operatorname{con}\left(A_{1}\right)=k$, it means that for each $1 \leq j \leq k$, the functional $f \mapsto f^{(j)}(0)$ is in $\Gamma_{1}$. Therefore each functional $g \mapsto g^{(j)}(0)$ is in $\Gamma_{2}$, and 
$\operatorname{con}\left(A_{1}\right) \leq \operatorname{con}\left(A_{2}\right)$. Finally if ord $\left(A_{1}\right)=n$, it means there is some $\Lambda_{1}$ in $\Gamma_{1}$ of the form (3.2) with $a_{n} \neq 0$; it follows that $\operatorname{ord}\left(A_{1}\right) \leq \operatorname{ord}\left(A_{2}\right)$.

Summarizing, we have shown:

Proposition 3.4. If $\phi: V_{1} \rightarrow V_{2}$ is an injective holomorphic map from one cusp to another, then $\operatorname{cod}\left(V_{1}\right) \leq \operatorname{cod}\left(V_{2}\right), \operatorname{con}\left(V_{1}\right) \leq \operatorname{con}\left(V_{2}\right)$ and $\operatorname{ord}\left(V_{1}\right) \leq \operatorname{ord}\left(V_{2}\right)$.

Now, if $P$ is a cusp point in a petal $V_{1}$, and one surrounds $P$ by a decreasing sequence of open sets $U_{n}$, then with each $U_{n}$ there is a cusp algebra with a codimension, contact and order, and there is an inclusion map from each $U_{n+1}$ to $U_{n}$. By Proposition 3.4, the positive integers $\operatorname{cod}\left(U_{n}\right), \operatorname{con}\left(U_{n}\right)$ and $\operatorname{ord}\left(U_{n}\right)$ must decrease as $U_{n}$ shrinks. Therefore, at some point they must stabilize, and for all sufficiently small neighborhoods of $P$, the cusp algebras have the same codimension, contact and order. We shall call these $(\operatorname{cod})(P),(\operatorname{con})(P)$ and $($ ord $)(P)$ respectively, and say $P$ is a simple cusp point if $\operatorname{con}(P)=1$.

\section{Equivalence of simple cusps}

We shall say that two points $P_{1}$ and $P_{2}$ in the petals $V_{1}$ and $V_{2}$ are locally equivalent if there is a neighborhood $U_{1}$ of $P_{1}$ in $V_{1}$, and a neighborhood $U_{2}$ of $P_{2}$ in $V_{2}$, and a biholomorphic homeomorphism $F$ from $U_{1}$ onto $U_{2}$ that maps $P_{1}$ to $P_{2}$.

Theorem 4.1. Two simple cusp points are locally equivalent if and only they have the same codimension.

Proof: Necessity follows from Proposition 3.4. To prove sufficiency, let $\pi_{1}$ be a primitive of the first algebra, and $\pi_{2}$ a primitive of the second algebra. By Proposition 2.2, it is sufficient to prove that there are neighborhoods $W_{1}$ and $W_{2}$ of the origin, and a univalent map $\phi: W_{1} \rightarrow W_{2}$ that maps 0 to 0 and such that

$$
\pi_{2} \circ \phi=\pi_{1} .
$$

Each $\pi_{r}$ has a square root $\chi_{r}$ in a neighborhood of 0 , and each $\chi_{r}$ is locally univalent (because $\pi_{r}$ is of order 2). So define

$$
\phi:=\chi_{2}^{-1} \circ \chi_{1}
$$

on a suitable neighborhood $W_{1}$, and (4.2) is satisfied.

How can we globally parametrize simple cusp algebras? We shall show that there is an essentially unique primitive with all its even Taylor coefficients (except for the second) zero. By $\hat{\pi}(k)$ we mean the $k^{\text {th }}$ Taylor coefficient at 0 . 
Lemma 4.3. Every simple cusp algebra of codimension $n+1$ has a primitive $\pi$ such that $\hat{\pi}(2 k)=0$ for all $2 \leq k \leq n$. Moreover, $\pi$ is unique up to $O\left(z^{2 n+2}\right)$.

Proof: (Existence): Let $\chi_{1}$ be any primitive. Define

$$
\chi_{2}:=\chi_{1}-\hat{\chi}_{1}(4)\left(\chi_{1}\right)^{2} .
$$

Proceed inductively, with

$$
\chi_{k}:=\chi_{k-1}-\hat{\chi}_{k-1}(2 k)\left(\chi_{k-1}\right)^{k} .
$$

Then let $\pi=\chi_{n}$.

(Uniqueness): Suppose $\pi$ and $\chi$ are both primitives with their even Taylor coefficients, starting at 4, vanishing. By Proposition 2.2, we have

$$
\chi=c_{1} \pi+c_{2} \pi^{2}+\cdots+c_{n} \pi^{n}+O\left(z^{2 n+2}\right) .
$$

By looking at the coefficient of $z^{2}$ we see $c_{1}=1$. Now looking at the coefficients of $z^{4}, z^{6}, \ldots, z^{2 n}$ in order, we see that $c_{2}=0=c_{4}=\cdots=$ $c_{n}$.

So every algebraic connection of dimension $n+1$ supported at the origin is the annihilator of an algebra generated locally by a unique primitive

$$
\pi(z)=z^{2}+\alpha_{1} z^{3}+\alpha_{2} z^{5}+\cdots+\alpha_{n} z^{2 n+1} .
$$

Let us denote the codimension $n+1$ subalgebra of $O(\mathbb{D})$ with primitive (4.4) by $A\left(\alpha_{1}, \ldots, \alpha_{n}\right)$, and let $V\left(\alpha_{1}, \ldots, \alpha_{n}\right)$ denote the corresponding petal $h(\mathbb{D})$, where $h$ holizes $A\left(\alpha_{1}, \ldots, \alpha_{n}\right)$.

Two cusps $V_{1}$ and $V_{2}$ are globally equivalent if there is a biholomorphic homeomorphism $\phi$ from $V_{1}$ onto $V_{2}$. If each $V_{r}$ is holized by $h_{r}: \mathbb{D} \rightarrow V_{r}$, then by $[\mathbf{1}$, Theorem 3.1], this occurs if and only if there is a map $\psi: \mathbb{D} \rightarrow$ $\mathbb{D}$ such that $\psi_{*}$ maps the first connection to the second. As the only selfmaps of the disk that leave the origin invariant are rotations, this is rather restrictive.

Theorem 4.5. The cusps $V\left(\alpha_{1}, \ldots, \alpha_{n}\right)$ and $V\left(\beta_{1}, \ldots, \beta_{n}\right)$ are isomorphic if and only if there is a unimodular constant $\tau$ such that $\beta_{j}=$ $\tau^{2 j-1} \alpha_{j}$ for $1 \leq j \leq n$.

As a corollary we have

Corollary 4.6. The moduli space of all simple cusps of codimension $n+1$ is $\mathbb{R}^{+} \times \mathbb{C}^{n-1}$. 


\section{Embedding}

The purpose of this section is to prove that every simple cusp algebra $A$ contains a pair of functions $h_{1}, h_{2}$ such that the pair holizes the algebra (which is equivalent to saying that polynomials in $h_{1}$ and $h_{2}$ are dense in $A$ in the topology of uniform convergence on compacta).

For the rest of this section, $A$ will be a fixed simple cusp algebra of codimension $n+1$.

Lemma 5.1. For every $\alpha$ on $\mathbb{D} \backslash\{0\}$, there is a function $\psi_{\alpha}$ in $A$ that has a single simple zero at $\alpha$, and no other zeroes on $\mathbb{D}$.

Proof: Consider functions of the form $(z-\alpha) e^{h(z)}$. In order to be in $A$, $h$ must satisfy $n+1$ equations on its first $2 n+1$ derivatives at 0 . This system is triangular, so can be solved with $h$ a polynomial.

Lemma 5.2. If $f$ is in $A$ and $f$ has no zeroes on $\mathbb{D}$, then $1 / f$ is in $A$.

Proof: Consider the vector space obtained by adjoining $1 / f, 1 / f^{2}, \ldots, 1 / f^{k}$ to $A$. Since $A$ is of finite codimension in $O(\mathbb{D})$, for some $k$ there is a linear relation

$$
1 / f^{k}=\sum_{j=1}^{k-1} c_{j} 1 / f^{j}+A .
$$

Multiplying both sides by $f^{k-1}$, we get that $1 / f$ is in $A$.

Lemma 5.3. If $f \in A$ and $f(\alpha)=0$ for some $\alpha \in \mathbb{D} \backslash\{0\}$, then $f / \psi_{\alpha}$ is in $A$.

Proof: Let $\Gamma$ be the connection at 0 such that $A=\Gamma^{\perp}$. Consider the algebra

$$
A^{\prime}:=\left\{f \in O\left(\frac{|\alpha|}{2} \mathbb{D}\right): \Lambda(f)=0, \forall \Lambda \in \Gamma\right\} .
$$

Then $\psi_{\alpha}$ is in $A^{\prime}$, so by Lemma $5.2,1 / \psi_{\alpha}$ is in $A^{\prime}$. Therefore $f / \psi_{\alpha}$ is in $A^{\prime}$, and is therefore annihilated by every functional in $\Gamma$. But $f / \psi_{\alpha}$ is also in $O(\mathbb{D})$, so it is in $A$ as required.

Theorem 5.4. There exists a map $h: \mathbb{D} \rightarrow \mathbb{C}^{2}$ that holizes $A$.

Proof: By applying Lemma 5.3 repeatedly, one can find a primitive $h_{1}$ of $A$ that has no zeroes in $\mathbb{D} \backslash\{0\}$. Define

$$
h_{2}(z):=z\left(h_{1}(z)\right)^{n+1} .
$$


Consider the algebra $A^{\prime}$ that is the closure (in the topology of uniform convergence on compacta) of polynomials in $h_{1}$ and $h_{2}$. By $[\mathbf{1}$, Theorem 4.2], the algebra $A^{\prime}$ is of finite codimension, and it is a cusp algebra, because $h(z)=\left(h_{1}(z), h_{2}(z)\right)$ is one-to-one away from the origin. If $A^{\prime} \neq A$, then it is contained in a maximal proper subalgebra $A^{\prime \prime}$ of $A$, which must have codimension $n+2$, and therefore order $2 n+3$. But $A^{\prime \prime}$ contains $h_{2}$ and $h_{1}^{n+1}$, and these functions have order $2 n+2$ and $2 n+3$ respectively at the origin. So there can be no linear relation in $A^{\prime \prime}$ between the derivatives at the origin of order $(2 n+2)$ and $(2 n+3)$, and so the order of $A^{\prime \prime}$ must actually be at most $2 n+1$.

\section{References}

[1] J. Agler And J. E. MC CARThy, Hyperbolic algebraic and analytic curves, Indiana Univ. Math. J. 56(6) (2007), 2899-2933.

[2] T. W. Gamelin, Embedding Riemann surfaces in maximal ideal spaces, J. Functional Analysis 2 (1968), 123-146.

[3] G. Knese, Function theory on the Neil parabola, Michigan Math. J. 55(1) (2007), 139-154.

[4] V. I. Paulsen An D. Singh, Modules over subalgebras of the disk algebra, Indiana Univ. Math. J. 55(5) (2006), 1751-1766.

Jim Agler:

Department of Mathematics

University of California, San Diego (UCSD)

La Jolla, California 92093-0112

USA

E-mail address: jagler@ucsd.edu

John E. McCarthy:

Mathematics Department

Washington University

St. Louis, Missouri 63130

USA

E-mail address: mccarthy@math.wustl.edu

Rebut el 14 de setembre de 2007. 